\title{
Cardiovascular risk assessment by FRS and SCORE in Iranian adult population
}

\author{
Alipasha Meysamie ${ }^{1 *}$, Fereshteh Salarvand ${ }^{2}$, MirHojjat Khorasanizadeh ${ }^{3}$, Reza Ghalehtaki ${ }^{4}$, Mahsa Eskian ${ }^{3}$, \\ Saeed Ghodsi ${ }^{3}$, Shirin Ghalehtaki ${ }^{5}$, Mehrshad Abbasi ${ }^{6}$, Koroush Etemad $^{7}$, Fereshteh Asgari ${ }^{7}$ \\ and Alireza Esteghamati ${ }^{6}$
}

\begin{abstract}
Background: Handling the growing epidemic of coronary heart disease in developing nations hinges on primary prevention, which logistically requires directing preventive interventions to those at the highest risk. Therefore, implementing cardiovascular risk assessment profiles is crucial to distinguish high risk groups who truly need extensive preventive measures. We aimed to draw a picture of the cardiovascular risk profiles in the Iranian adult population for the first time.
\end{abstract}

Methods: Demographic, anthropometric, and laboratory data as well as blood pressure and smoking status of 3944 subjects participating in the 2011 national surveillance of risk factors for non-communicable diseases were used to calculate the mean estimated risk of coronary artery disease and the relative frequency of low-, medium- and highrisk subjects based on FRS and SCORE indices in general population as well as different age, sex, and residence subgroups.

Results: The average 10-year risk of coronary artery disease (FRS) and 10-year risk of fatal coronary and cerebrovascular accidents (SCORE) in the 25 to 64 year-old population was 13.82 and 0.72 respectively. The relative frequency of the intermediate- and high- risk subjects was 25.8 and $22.6 \%$ based on FRS and 9.2 and $1.8 \%$ based on SCORE respectively. Average FRS and SCORE were significantly higher among men than women, but were not significantly different among urban and rural residents.

Conclusions: A significant proportion of the Iranian population, based on FRS model, will be at moderate to high risk of coronary events in the next 10 years. Urgent preventive plans are needed the national level.

Keywords: Risk factors, Risk assessment, Coronary heart disease, Cardiovascular disease, SCORE, FRS, Iran

\section{Background}

Coronary heart disease (CHD) is characterized by presence of atherosclerosis in epicardial coronary arteries and subsequent disruption of myocardial blood flow, which according to the extent of obstruction and pace of its progression could result into myocardial infarction (MI) and death. CHD is one of the most prevalent causes of death in many communities. It is responsible for about one-third of deaths among adults older than 35 in the United States. In 2008, among noncommunicable diseases (NCDs), CHD was the leading

\footnotetext{
* Correspondence: meysamie@tums.ac.ir

${ }^{1}$ Department of Community and Preventive Medicine, Faculty of Medicine,

School of Medicine, Tehran University of Medical Sciences, Tehran, Iran

Full list of author information is available at the end of the article
}

cause of death $(48 \%)$ in low-middle income countries [1]. Cardiovascular diseases including acute myocardial infarction, unstable angina, sudden cardiac death, and stroke has been reported to have a high and rising burden in Iran and claim lives of men and women even in young ages [2]. CHD is one of the main causes of mortality and morbidity in Iranian population, and according to the available evidence, it is responsible for about $50 \%$ of total annual deaths [3]. Despite lack of accurate data, some evidences show that the prevalence of CHD in Iran is currently $21 \%$, and is increasing each year [4]. While age-adjusted mortality rate of CHD is decreasing in developed countries, on average, this rate has increased by $20-45 \%$ in Iran [5]. 
The growing epidemic of CHD in Iran needs to be addressed urgently. Although there has been a major progress in therapeutic techniques for patients with symptomatic CHD, many initial events are fatal, and the treatment options are more palliative than curative in nature. Consequently, a truly decisive effect on the CHD epidemic will require primary prevention of incident disease, considering that most cardiovascular risk factors are modifiable. Measures such as life style modifications mainly involving diet and physical activity, taking medications like statins and aspirin, and angiographic or surgical interventions could moderate these risk factors [6].

Most studies emphasize on the benefits of risk factor modifying interventions, but consider these interventions to be cost-effective only in medium- to high-risk groups [7]. Widespread inclusion of such programs into care routines of healthcare centers and primary-care physician offices is associated with significant costs and requires rigorous measures. Therefore, the use of risk assessment models which provide the opportunity to direct the preventive measures to the at-risk population is unavoidable.

Aiming to identify the at-risk individuals, various global risk assessment profiles, derived from longitudinal cohort studies, have been optimized in the past 30 years. A wellknown risk profile, recommended in clinical guidelines, is Framingham Risk Score (FRS), which predicts the 10-year risk of incident CHD - including fatal and non-fatal MI, unstable angina and stable anginas - based on the level of individual risk factors. Another model mostly adopted in western European countries is the Systematic coronary Risk Evaluation (SCORE) model, which predicts the 10-year risk of incident fatal atherosclerotic cardiovascular events, including myocardial and cerebral infarction [8]. These two risk assessment profiles have been validated in different geographic regions using reliable statistical methods [9].

Since in our country, Iran, there is no community-based evidence regarding the risk of CHD and fatal cardio- or cerebro-vascular events according to FRS or SCORE models at the national level, we aimed to assess the overal risk of CHD and fatal cardio- or cerebro-vascular events in 25-64 year-old Iranian subjects in this study. Knowing the 10-year risk of CHD and fatal cardio- or cerebrovascular events is helpful in planning preventive measures for populations at the highest risk, especially in the resource-limited settings.

\section{Methods}

\section{Study population}

In the Surveillance of Risk Factors of Non-communicable Diseases (SuRFNCD) in 2011, a total of 11,867 Iranian individuals aged 6-70 years (excluding the nomadic tribes who are not covered by the Iranian postal service) were surveyed using Random complex sampling. Among the
25-64 years-old included subjects, 4759 individuals consented for blood sampling. After the re-evaluation of data and laboratory samples, and exclusion of pregnant subjects, ultimately 3944 individuals with complete demographic, laboratory and smoking status information entered the statistical analysis as the final sample. More information regarding the sampling method has been previously described elsewhere [10].

\section{Questionnaires, measurements and definitions}

According to the Surveillance of Risk Factors of Noncommunicable Diseases (SuRFNCD) international program, STEPS questionnaires of world health organization (WHO) were subjected to the participants in 6 sections including demographic information, diet, physical activity, tobacco smoking, history of hypertension and diabetes. Laboratory examination of blood samples was performed for variables such as high density lipoprotein (HDL), total serum cholestrol, triglyceride and glucose. All tests were performed using quality controlled commercial kits (Pars Azmun, Karaj, Iran) distributed by the center for disease control (CDC) reference laboratory. Inflexible measurement tapes and portable digital scales were respectively used to measure height and weight. Height and weight were measured with subjects standing without socks and shoes and dressed lightly. Blood pressure was measured using calibrated sphigmomanometer on three occasions spaced for $5 \mathrm{~min}$, and the mean value was used. Waist circumference was measured mid-way around the direct line that connects the lower costal margin and anterior-superior iliac spine at the end of normal expiration, with arms extended and alligned with body.

\section{Statistical analysis}

Weighting of the data was initially performed in order to describe the overall population regarding the stratified cluster sampling method being applied. After excluding duplicate, missing and outlier data, weighting was performed based on the 2011 official national population census and strata of provinces, residence (urban or rural), gender (male or female), and age groups (10-year categories 25-34, 35-44, 45-54, and 55-64). The minimum and maximum weights were 99,951 for $35-44$ year old male indiviuals living in urban areas of Bushehr province and 315 for 55-64 year old female indivuals living in rural areas of Ilam province. The median weight was 6880 . The total extrapolated population size was $42,287,987$. Thereafter, the data were analysed using complex sample survey method opting SPSS version 20 for Windows. The 10-year risk of coronary heart diseases and the 10-year risk of fatal cardiovascular events were calculated using FRS and SCORE functions respectively. Then the subjects were categorized into low-, intermediate- and high-risk groups based on their 
FRS and SCORE risk values. The mean score and relative frequency of any given gender, residential and age strata in each risk group were calculated with $95 \%$ confidence interval. The detailed methods of calculation of FRS and SCORE have been explained elsewhere $[8,11]$.

\section{Research ethics and patient consent}

This study was approved by institutional review board of the Center for Disease Control of Iranian Health Ministry. All steps of this study were conducted in accordance with the guidelines and standards laid down in the latest revision of the Declaration of Helsinki. For confidentiality reasons, patients' names were not used in the study, and the allocated codes were used instead. Written informed consent was provided by all participants.

\section{Results}

\section{The 10-year risk of CHD based on FRS}

The mean age of studied subjects was 38.40 years $(\mathrm{CI} 95 \%=37.6-39.21) .49 .7 \%$ were male and $68.8 \%$ were urban residents. Of the participants, $40.6 \%$ were in the $25-34,28.5 \%$ in $35-44,19.9 \%$ in $45-54$, and finally $11 \%$ in the 55-64 years age group.

The mean 10-year risk of CHD based on FRS in the studied population was $13.82(\mathrm{CI} 95 \%=13.47-$ 14.17). The relative frequency of low-, medium- and high-risk subjects in the studied population was $51.6 \%$ (49.9-53.3), 25.8\% (24.1-27.7) and 22.6\% (21.2-24.0), respectively (Table 1 ).

The mean Framingham Risk Score was significantly higher in men than women; 15.95 (15.37-16.53) for men vs. 11.54 (11.19-11.88) for women. There was no statistically significant difference in FRS between urban and rural residents $(P=0.1)$. The mean FRS increased significantly by increasing age to reach 31.36 in the 55-64 age group, compared with mean FRS of 5.55 in the 25-34 age group.

The frequency of high-risk subjects in men was significantly higher than women, with $29.7 \%$ (27.3-32.1) for men vs. $14.9 \%(13.7-16.3)$ for women. However, there was no significant difference between urban and rural residents. In higher age groups, the frequency of highrisk subjects was significantly higher than lower age groups; e.g. $75.4 \%(72.1-78.4)$ in the $55-64$ age group, compared with $0.8 \%(0.3-1.9)$ in the $25-34$ age group.

\section{The 10-year risk of fatal cardio- and cerebro-vascular events based on SCORE}

The mean 10-year risk of fatal cardio- and cerebrovascular events based on SCORE in the studied population was $0.72(\mathrm{CI} 95 \%=0.68-0.75)$. The frequency of low-risk, medium-risk and high-risk subjects in the studied population was $89.0 \%$ (88.0-89.9), 9.2\% (8.3-10.2) and $1.8 \%(1.5-2.2)$ respectively (Table 2$)$.
The mean score calculated based on SCORE was significantly higher in men than women; with 1.05 (0.99-1.11) for men vs. $0.36(0.33-0.38)$ for women. There was no statistically significant difference in SCORE between urban and rural residents $(P=0.09)$. The mean SCORE increased significantly by increasing age to reach 3.12 (2.89-3.35) in the 55-64 age group, compared with mean SCORE of 0.04 (0.043-0.0544) in the 25-34 age group.

The frequency of high-risk subjects in men was significantly higher than women, with $3.1 \%(2.5-3.9)$ for men vs. $0.4 \%(0.2-0.6)$ for women. However, there was no significant difference between urban and rural residents. In higher age groups, the frequency of high-risk subjects was significantly higher than lower age groups; e.g. $12.4 \%(10.2-15.0)$ in the 55-64 age group, compared with $0 \%$ in the $25-34$ age group.

\section{Discussion}

The main goal of this study was to perform a cardiovascular risk assessment of the Iranian adult population. In order to achieve this goal, the 10-year risk of incident coronary artery disease based on Framingham Risk Score (FRS) and the 10-year risk of incident fatal atherosclerotic events including coronary artery disease and cerebrovascular accidents based on Systematic COronary Risk Evaluation (SCORE) were calculated in the 25-64 years-old population.

As was thoroughly explained in the Results section, mean FRS in Iranian population is considerably high (13.82), and more than $20 \%$ of the Iranian population which equals to 13.08 million people are in the high-risk group (FRS $\geq 20$ ) for coronary artery disease. FRS was higher in older age groups, and in men compared with women. In contrast to expectations, there was no significant difference in mean FRS and frequency of high-risk individuals between urban and rural residents. Previously, the discrimination power of FRS for prediction of cardiovascular mortality at 5 and 10 years have been shown in Iranian urban population in Tehran Lipid and Glucose prospective study [12, 13].

The relatively high mean FRS has been pointed out earlier by other authors in specific Iranian populations. Among these examples are the mean FRS figures reported as high as $10.2 \pm 7.1$ in postmenopausal women by Eshtiaghi et al. [14], and $13.0 \pm 8$ in men and women with at least one cardiovascular risk factor by Nematy et al. [15].

In the study of Ford et al. in United States, the relative frequency of low-, medium- and high-risk groups based on FRS was $81.7,15.5$ and $2.9 \%$ respectively [16]. In this study, the mean FRS was higher in older age groups, and in men compared with women; as the relative frequency of high- and medium-risk subjects were respectively 5.3 and $38.7 \%$ in men and 0.9 and $4.3 \%$ in women. Although this general pattern is consistent with the results of our 


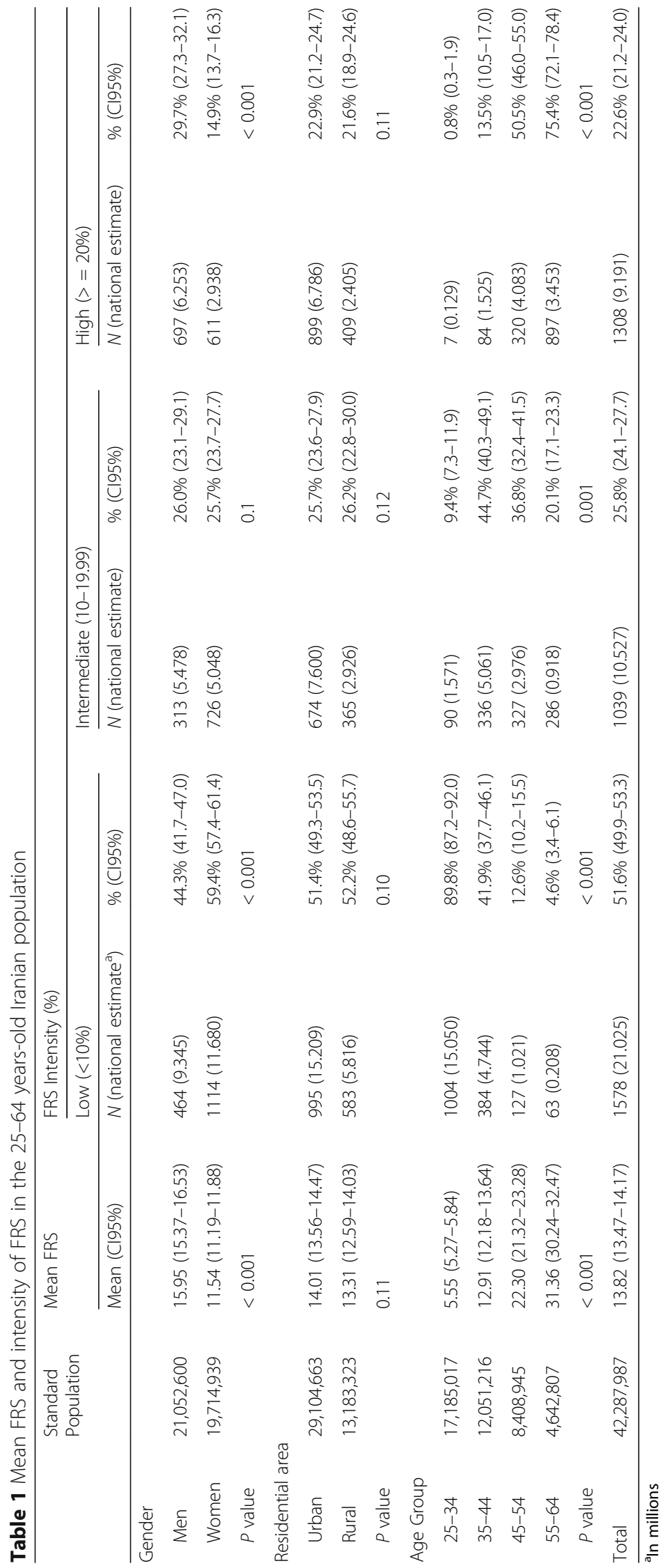




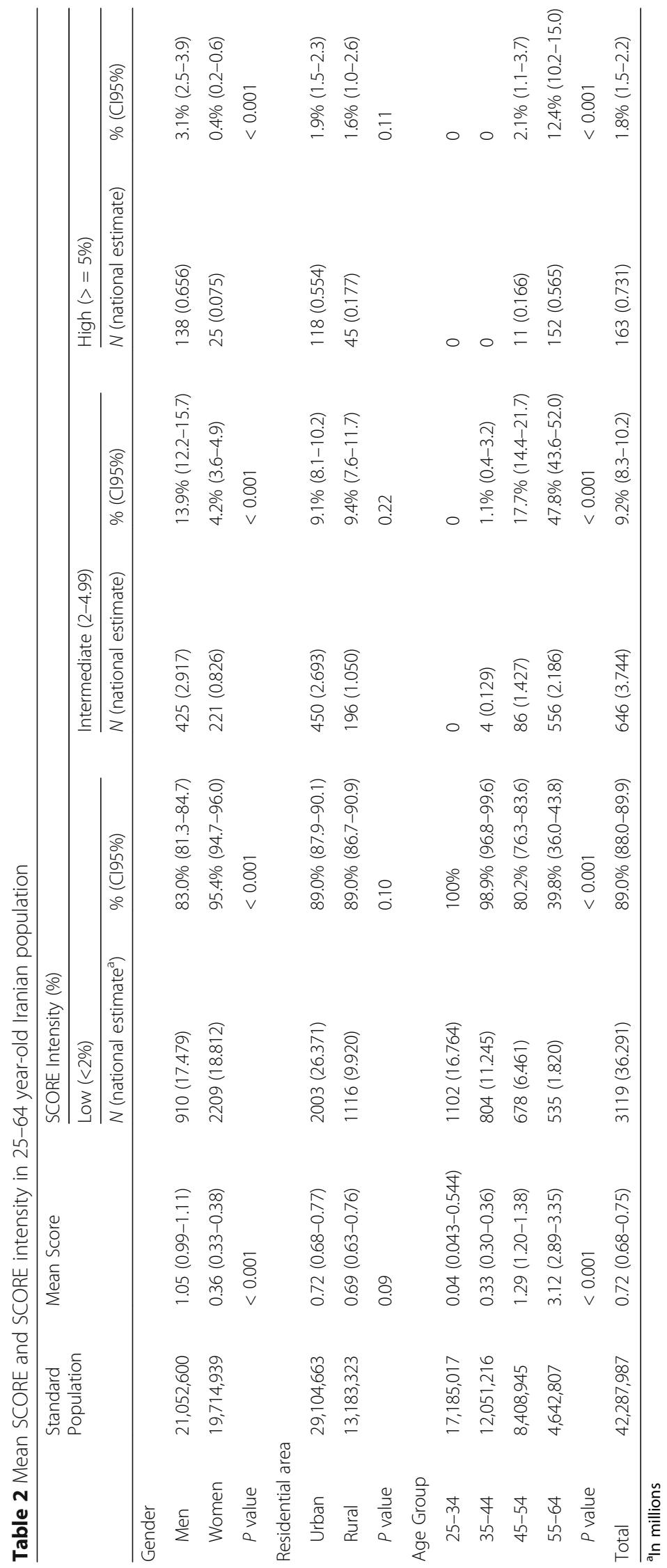


study, but in our study the frequency of medium- and high-risk subjects were much higher in the 2564 year-old Iranian population. One reason could be the difference between study populations. Regarding risk assessment, best results can be achieved when the population being evaluated is similar to the reference population of the applied risk index. The reference population for FRS index was socio-economically advantaged Caucasian subjects; therefore this index over-estimates the risk in African, Native American and Asian ethnic groups [17]. In agreement with this statement, in a systematic review by Brindle et al., the predicted to observed ratios based on FRS ranged from an underprediction of 0.43 (95\% CI 0.27 to 0.67 ) in a high-risk population to an overprediction of 2.87 (95\% CI 1.91 to 4.31 ) in a lower-risk population, suggesting that the performance of FRS varies considerably between populations [18]. In another study, Chia et all showed that cardiovascular disease (CVD) risk in a multiethinic east Asian society calculated by FRS model could only make a moderate discrimination, with an area under the receiver operating characteristic curve (AUC) of 0.63 [19]. On this basis, it seems that using a model derived from characteristics of the Iranian population could predict the 10-year risk of coronary artery disease with more accuracy. But, until then FRS may act as an alternative risk assessment tool.

The mean SCORE index in Iranian population is relatively low (0.72), and based on this index about $2 \%$ of the total 25-64 year old population which equals to 730,000 people are categorized in the high-risk group (SCORE $\geq 5$ ). As for FRS, the mean SCORE was higher in older age groups, and in men compared with women, and there was no significant difference in mean SCORE and frequency of high-risk individuals between urban and rural residents.

Our 10 year risk estimates of fatal cardio- or cerebrovascular events calculated using SCORE model were far lower than those of europian countries. One explanation for this difference is that we used this model for all above 25 year old population, whereas SCORE model was developed according to the mortality data of 4075 year old population in mostly western Europian countries [8]. Interestingly, in our study, the prevalence of high risk subjects was 5.6 and $12.4 \%$ among over 45 and 55 year old population, respectively. There are no reports of risk stratification using SCORE in middleeastern countries to make comparisons.

In comparison between FRS and SCORE models, Barroso et al. showed that among middle to old age Spanish population SCORE performed better in predicting CVD risk discrimination. In that study, 18.3 and $9.2 \%$ of the population were put into high risk category based on
FRS and SCORE, respectively [20]. The lower estimates calculated by SCORE compared to FRS in our study and others in part returns to the aim of these models; SCORE was developed to predict only fatal events while the mission of FRS was to predict both non fatal and fatal cardiovascular events. Aside from this explanation other studies have shown that FRS usually estimates a higher risk inferred by each individual compared with SCORE [21].

Among the results it was noted that FRS and SCORE tend to markedly increase with age. It seems that the most prominent rationale for this could be the augmented insulin resistance and mean blood preasure as age increases. Actually, these two factors contribute most to the positive correlation between age and cardiovascular risk [22].

Moreover, in this study we showed that not only the mean 10-year risk of coronary artery disease based on FRS and SCORE indices is significantly higher in men than in women, but also the frequency of high-risk subjects is significantly higher among men. This difference could originate from the effect of sex hormones and their considerable protective role in women, as in the study of Eshtiaghi et al. in women aged 20-76 years was shown that mean FRS in the post-menopausal group of participants was significantly higher that the pre-menopausal group (10.2 vs. 1.6) [14].

In contrary to our expectations, there was no significant difference in mean FRS and SCORE and frequency of medium- to high-risk subjects between urban and rural residents in this study. Assuming that urban and rural residents are much different in diet and physical activity habbits, one might speculate that rural residents face a lower risk compared with urban residents, which is not true based on our results. Actually, there was no significant difference between urban and rural residents in many of the cardiovascular risk factors incorporated in FRS and SCORE models both in our study (data not shown) and previous studies [23]. These findings indicate that future preventive interventions should equally cover both urban and rural population.

Calculating 10-year risk of cardiovascular diseases through well-known global cardiovascular risk assessment models including FRS and SCORE is widely performed all over the world and is recommended for all adults. However, there are some limitations to these models. For instance, FRS may under- or over-estimate the risk in certain populations, its 10-year scope is relatively short, it mostly focuses on myocardial infarction and coronary artery disease-attributed death, and ignores family history. Regarding SCORE, one of the most important limitations is ignoring triglyciride and fibrinogen levels, and also family history of early-onset cardiovascular disease [8]. Categorizing the subjects into low-risk $(<10 \%)$, 
medium-risk (10-20\%) and high-risk (>20\%) groups could be biased by up to $37 \%$, especially in women and young adults. In order to address these limitations, one of the proposed solutions is to use life-time risk assessment models, as a considerable proportion of subjects with low 10-year risk, have a life-time risk of more than 39\% [24]. Moreover, in order to further categorize intermediate risk individuals, more novel risk factors such as Lp-PLA ${ }_{2}$, LDL-P, fibrinogen, Lp-a, small dense low density lipoprotein (LDL), plasminogen activator inhibitor (PAI-1), IL-6, CRP, coronary artery calcification (CAC) and carotide intima-media thickness can be used [25].

\section{Limitations}

Considering the large population of this study, the probability of non-cooperation of participants in filling out the questionnaires and providing blood samples and anthropometric measurements were among the limitations of our study. Therefore, the most important limitation of this study is the high amount of missing data both during data collection and data entry into the statistical software. Actually the authors faced a lot of missing, duplicate and outlier values. To moderate the effect of this problem, the duplicate and outlier data were cleared as much as possible, and in the final statistical analysis only the cleared data were weighted. Another limitation that severely impacts our conclusions is our cross-sectional design of the study. In fact, we can not say for sure whether the high risk subjects discriminated by FRS and SCORE models will be affected by cardiovascular accidents in future or not.

\section{Conclusion}

In this study we showed that mean FRS in the 2564 years-old Iranian population is markedly high, and more than $20 \%$ of the population are in the high-risk group. The mean risk and frequency of high-risk subjects were lower based on the SCORE index. These results show that in the upcoming years the Iranian population is going to be in great risk for cardiovascular events, and more rigorous primary prevention programs are urgently needed at the national level. However, when comparing these risk values with those of other populations, it should be noted that differences between the studied population and the reference population of the risk assessment model in use, increase the error of measurement. Therefore we propose that a risk assessment model derived from the Iranian population characteristics be designed and validated in future studies, and its power in predicting the risk of cardiovascular events be compared with available models.

\section{Abbreviations}

AUC: Area under curve; CAC: Coronary artery calcification; CDC: Center for disease control; CHD: Coronary heart disease; CVD: Cardiovascular disease; FRS: Framingham Risk Score; HDL: High density lipoprotein; LDL: Low density lipoprotein; MI: Myocardial Infarction; NCDs: Non-communicable diseases; SCORE: Systematic COronary Risk Evaluation; STEPS: STEPwise approach to Surveillance; SuRFNCD: Surveillance of Risk Factors of Non-communicable Diseases

Acknowledgements

Not applicable.

\section{Funding}

In this paper the data were used from the 2011 national surveillance of risk factors for non-communicable diseases which was sponsored by the Iranian Health Ministry and Center for Disease Control. This research in particular received no specific grant from any funding agency in the public, commercial, or not-for-profit sectors.

\section{Availability of data and materials}

Please contact corresponding author for data requests.

\section{Authors' contributions}

AM, FS, RG and SG contributed to the conception or design of the work. AM, $F S, R G, S G, M A, K E, F A$, and $A E$ contributed to the acquisition, analysis, or interpretation of data for the work. MK, ME, FS and RG drafted the manuscript. AM critically revised the manuscript. All gave final approval and agree to be accountable for all aspects of work ensuring integrity and accuracy.

Ethics approval and consent to participate

Our study was reviewed and approved by ethics committee, deputy of research, Medical school, Tehran University of Medical Sciences.

Consent for publication

Not applicable.

\section{Competing interests}

The authors declare that they have no competing interests

\section{Publisher's Note}

Springer Nature remains neutral with regard to jurisdictional claims in published maps and institutional affiliations.

\section{Author details}

'Department of Community and Preventive Medicine, Faculty of Medicine, School of Medicine, Tehran University of Medical Sciences, Tehran, Iran. ${ }^{2}$ Department of Dermatology, Razi Hospital, Tehran University of Medical Sciences, Tehran, Iran. ${ }^{3}$ School of Medicine, Tehran University of Medical Sciences, Tehran, Iran. ${ }^{4}$ Radiation Oncology Research Center, Cancer Institute, Tehran University of Medical Sciences, Tehran, Iran. ${ }^{5}$ School of Medicine, Ahvaz Jondishapour University of Medical Sciences, Ahvaz, Iran.

${ }^{6}$ Endocrinology and Metabolism Research Center (EMRC), Vali-Asr Hospital,

School of Medicine, Tehran University of Medical Sciences, Tehran, Iran.

${ }^{7}$ Center for Disease Control, Ministry of Health, Tehran, Iran.

Received: 24 March 2017 Accepted: 15 August 2017

Published online: 23 August 2017

\section{References}

1. Organization WH. Global Status Report on Non-Communicable Diseases 2010 [6/19/2016]. Available from: http://www.who.int/nmh/publications/ ncd_report2010/en/.

2. Talaei M, Sarrafzadegan N, Sadeghi M, Oveisgharan S, Marshall T, Thomas $\mathrm{GN}$, et al. Incidence of cardiovascular diseases in an Iranian population: the Isfahan cohort study. Arch Iran Med. 2013:16(3):138-44.

3. Hatmi ZN, Tahvildari S, Gafarzadeh Motlag A, Sabouri KA. Prevalence of coronary artery disease risk factors in Iran: a population based survey. BMC Cardiovasc Disord. 2007;7:32. 
4. Sepanlou SG, Kamangar F, Poustchi H, Malekzadeh R. Reducing the burden of chronic diseases: a neglected agenda in Iranian health care system, requiring a plan for action. Arch Iran Med. 2010;13(4):340-50.

5. Hadaegh F, Harati H, Ghanbarian A, Azizi F. Prevalence of coronary heart disease among Tehran adults: Tehran lipid and glucose study. East Mediterr Health J. 2009:15(1):157-66.

6. Capewell S, Ford ES, Croft JB, Critchley JA, Greenlund KJ, Labarthe DR. Cardiovascular risk factor trends and potential for reducing coronary heart disease mortality in the United States of America. Bull World Health Organ. 2010;88(2):120-30

7. Kamboj L, Oh P, Levine M, Kammila S, Casey W, Harterre D, et al. Cost effectiveness of a systematic guidelines-based approach to the prevention and management of vascular disease in a primary care setting. Int J Cardiol. 2016;203:893-9.

8. Conroy RM, Pyorala K, Fitzgerald AP, Sans S, Menotti A, De Backer G, et al. Estimation of ten-year risk of fatal cardiovascular disease in Europe: the SCORE project. Eur Heart J. 2003;24(11):987-1003.

9. Wannamethee SG, Shaper AG, Lennon L, Morris RW. Metabolic syndrome vs Framingham risk score for prediction of coronary heart disease, stroke, and type 2 diabetes mellitus. Arch Intern Med. 2005;165(22):2644-50.

10. Noshad S, Abbasi M, Etemad K, Meysamie A, Afarideh M, Khajeh E, et al. Prevalence of metabolic syndrome in Iran: a 2011 update. J Diabetes. 2017; 9(5):518-25.

11. D'Agostino RB Sr, Vasan RS, Pencina MJ, Wolf PA, Cobain M, Massaro JM, et al. General cardiovascular risk profile for use in primary care: the Framingham heart study. Circulation. 2008;117(6):743-53.

12. Bozorgmanesh $M$, Hadaegh $F$, Azizi F. Predictive accuracy of the 'Framingham's general CVD algorithm' in a middle eastern population: Tehran lipid and glucose study. Int J Clin Pract. 2011;65(3):264-73.

13. Khalili D, Hadaegh F, Soori H, Steyerberg EW, Bozorgmanesh M, Azizi F. Clinical usefulness of the Framingham cardiovascular risk profile beyond its statistical performance: the Tehran lipid and glucose study. Am J Epidemiol. 2012;176(3):177-86.

14. Eshtiaghi R, Esteghamati A, Nakhjavani M. Menopause is an independent predictor of metabolic syndrome in Iranian women. Maturitas. 2010;65(3):262-6.

15. Nematy M, Alinezhad-Namaghi M, Rashed MM, Mozhdehifard M, Sajjadi SS, Akhlaghi S, et al. Effects of Ramadan fasting on cardiovascular risk factors: a prospective observational study. Nutr J. 2012;11:69.

16. Ford ES, Giles WH, Mokdad AH. The distribution of 10-year risk for coronary heart disease among US adults: findings from the National Health and nutrition examination survey III. J Am Coll Cardiol. 2004; 43(10):1791-6

17. Dent TH. Predicting the risk of coronary heart disease I. The use of conventional risk markers. Atherosclerosis. 2010;213(2):345-51.

18. Brindle P, Beswick A, Fahey T, Ebrahim S. Accuracy and impact of risk assessment in the primary prevention of cardiovascular disease: a systematic review. Heart. 2006;92(12):1752-9.

19. Chia YC, Gray SY, Ching SM, Lim HM, Chinna K. Validation of the Framingham general cardiovascular risk score in a multiethnic Asian population: a retrospective cohort study. BMJ Open. 2015;5(5):e007324.

20. Barroso LC, Muro EC, Herrera ND, Ochoa GF, Hueros Jl, Buitrago F. Performance of the Framingham and SCORE cardiovascular risk prediction functions in a non-diabetic population of a Spanish health care centre: a validation study. Scand J Prim Health Care. 2010;28(4):242-8.

21. van der Heijden AA, Ortegon MM, Niessen LW, Nijpels G, Dekker JM Prediction of coronary heart disease risk in a general, pre-diabetic, and diabetic population during 10 years of follow-up: accuracy of the Framingham, SCORE, and UKPDS risk functions: the Hoorn study. Diabetes Care. 2009;32(11):2094-8.

22. Crouse JR 3rd, Bots ML, Evans GW, Palmer MK, O'Leary DH, Grobbee DE, et al. Does baseline carotid intima-media thickness modify the effect of rosuvastatin when compared with placebo on carotid intima-media thickness progression? The METEOR study. Eur J Cardiovasc Prev Rehabil. 2010;17(2):223-9.

23. Esteghamati A, Meysamie A, Khalilzadeh O, Rashidi A, Haghazali M, Asgari F, et al. Third national surveillance of risk factors of non-communicable diseases (SuRFNCD-2007) in Iran: methods and results on prevalence of diabetes, hypertension, obesity, central obesity, and dyslipidemia. BMC Public Health. 2009;9:167.
24. Relationship of atherosclerosis in young men to serum lipoprotein cholesterol concentrations and smoking. A preliminary report from the Pathobiological Determinants of Atherosclerosis in Youth (PDAY) Research Group. JAMA. 1990;264(23):3018-24.

25. Thompson A, Gao P, Orfei L, Watson S, Di Angelantonio E, Kaptoge S, et al. Lipoprotein-associated phospholipase a(2) and risk of coronary disease, stroke, and mortality: collaborative analysis of 32 prospective studies. Lancet (London, England). 2010;375(9725):1536-44.

\section{Submit your next manuscript to BioMed Central and we will help you at every step:}

- We accept pre-submission inquiries

- Our selector tool helps you to find the most relevant journal

- We provide round the clock customer support

- Convenient online submission

- Thorough peer review

- Inclusion in PubMed and all major indexing services

- Maximum visibility for your research

Submit your manuscript at www.biomedcentral.com/submit
) Biomed Central 